\title{
DN Term Type
}

National Cancer Institute

\section{Source}

National Cancer Institute. DN Term Type. NCI Thesaurus. Code C45774.

The NCl term type designation for a "display name". 